\title{
An introduction to the Babcock designed super dock blocks
}

\author{
George Kerr*, BEng, CEng, MRINA ${ }^{\mathrm{a}}$, Nancy Georgantzi, CEng, MRINA ${ }^{\mathrm{a}}$ \\ ${ }^{a}$ Babcock International Group - Energy and Marine Technology, Devonport, UK \\ *Corresponding author. Email: George.Kerr@babcockinternational.com
}

\begin{abstract}
Synopsis
This paper considers the issue of gaining access to the full extent of a vessel's bottom plating whilst it is dry docked. The current methods of secondary docking positions and block removals are reviewed and a new approach using jacks is presented. The development of an initial version of the new approach is described. This covers the elements of removal and replacement concept, general arrangement, limitations of the existing equipment, removal equipment and wood compression properties. These aspects are then brought together to create the requirements for the jacks. Operational experience on the first three dockings using the initial version at Babcock's Devonport site is discussed. The limitations of the initial approach are quantified and a fully developed solution which allows $100 \%$ block removal is presented. The paper concludes that the new approach of using jack-able super blocks provides benefits to both the shipyard and the ship operator. This project has been achieved through new research and the application of a sound knowledge of engineering and tolerances. The intellectual property of the paper is the subject of a pending patent.
\end{abstract}

Keywords: Dry dock; Super dock blocks; Dock blocks; Block removal; Block replacement; Jack

\section{Introduction}

Due to a challenging schedule required for the refitting of HMS Scott in 2013, Babcock (at Devonport Royal Dockyard) decided to investigate alternative approaches to dock block removal and replacement.

The first stage of the process involved developing a new approach to using existing equipment which allowed blocks to be removed in a significantly reduced timescale compared to the traditional methods previously in use.

This first stage can be deployed for around $75 \%$ of the blocks needed. However, some blocks require a higher load capacity.

As a second stage the arrangement was developed by designing stronger blocks which are sufficient to accept the load in all locations. These blocks are defined as super dock blocks and are currently being fabricated in Devonport. The super dock blocks allow every block to be removed using the new faster system. Furthermore, in many scenarios, they offer the potential to carry out hydrostatic testing of tanks whilst docked.

The intellectual property concerning these developments is the subject of a pending patent.

\section{Previous Situation}

The dock furniture in Devonport is traditionally used as shown below (Figure 1). The existing Type 1 dock blocks are reinforced concrete structures with steel tops and forklift holes. Each concrete block is used with steel wedges and two or three layers of oak timber to achieve the required height.

When any vessel is docked, the blocks on which she sits obstruct part of the keel, preventing access for survey and painting. The problem can be solved with either of the following two approaches:

1. There are two docking positions, which allow for different parts of the keel to be covered.

2. A method of removing blocks whilst the vessel is docked is used. About a fifth of the blocks are removed at a time.

In the first approach, the docking of a vessel is carried out in two phases. During the first phase, the vessel is positioned on the dock blocks and the exposed parts of the bottom are surveyed, blasted and painted. Phase one is complete when all the works in the exposed parts of the bottom have been finished. In phase two, the dock is flooded and the ship is fleeted forward by the distance of one block spacing. The dock is drained again and the remainder of the bottom can be accessed.

\section{Author's Biography}

George Kerr is a Principal Naval Architect with Babcock at Devonport Royal Dockyard. Since graduating from the University of Southampton he has worked in the small craft sector with CEproof Ltd. and during fourteen years with Babcock, held a variety of positions including Naval Architect on a major super-yacht build and Discipline Lead for Naval Architecture.

Nancy Georgantzi is a Senior Naval Architect with Babcock at Devonport Royal Dockyard. Since her graduation from the National Technical University of Athens she has worked in a number of dockyards and technical offices in Greece, dealing with commercial vessels of various types. During her six years in Babcock she has widened her experience working on Naval Ship design tasks. 
Babcock do use this approach for some dockings, but typically the disruption of undocking midway through a refit causes more expense than using method two. More typically, the docking position is alternated between each scheduled docking.

The second approach of block removal is generally followed in Devonport. It involves extracting the steel wedges from each dock block, which then allows the wooden stack to be dismantled. However, the wedges and the steel surfaces which they bear on corrode easily and over a short period of use the surfaces become uneven due to flaking corrosion. This makes the wedges difficult to remove. When this happens, it is necessary to split out the wood whilst it is still under load. This is a time consuming process, which can take between half a day to two weeks per block.

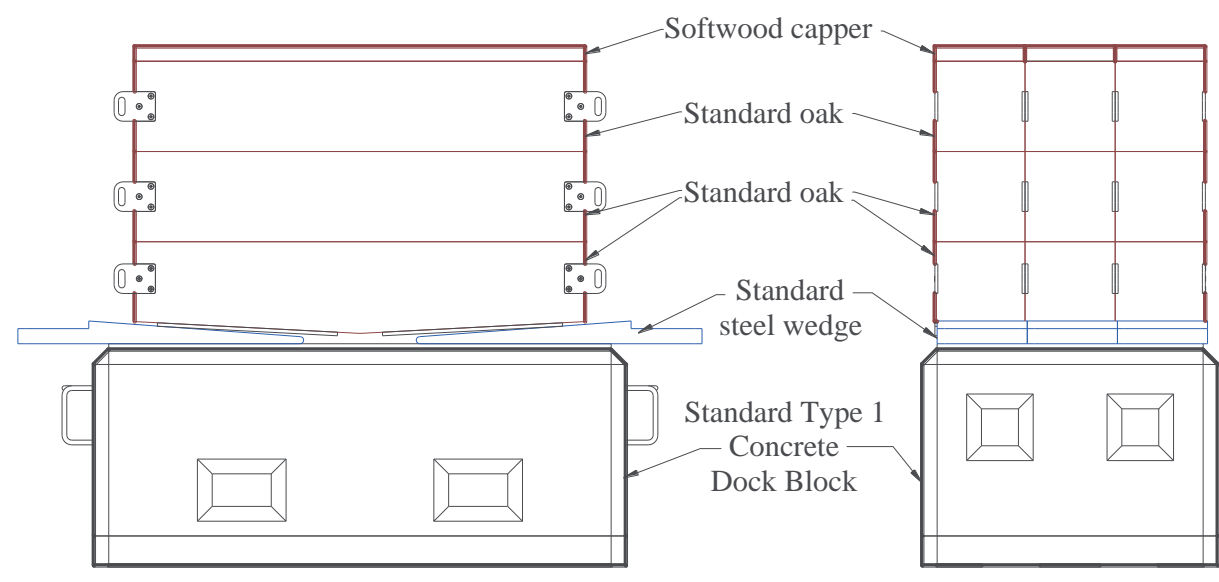

Figure 1: Traditional stack, Type 1 dock block

\section{Investigation of the new removal / replacement method}

A new process of dock block removal has been developed in Babcock that allows the removal of a block stack using a jacking arrangement. The concept of the new system requires two blocks stacked on top of each other as shown below (Figure 2).

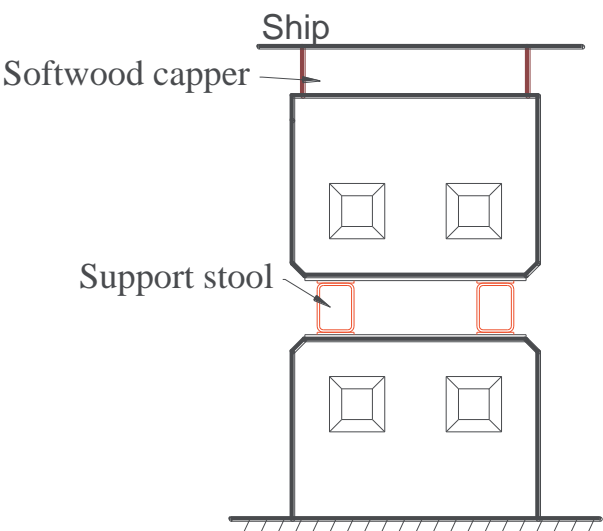

A

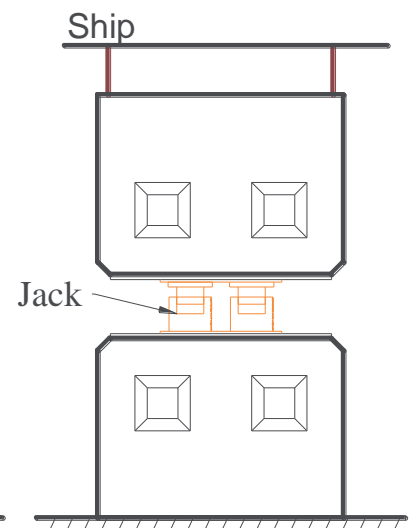

B

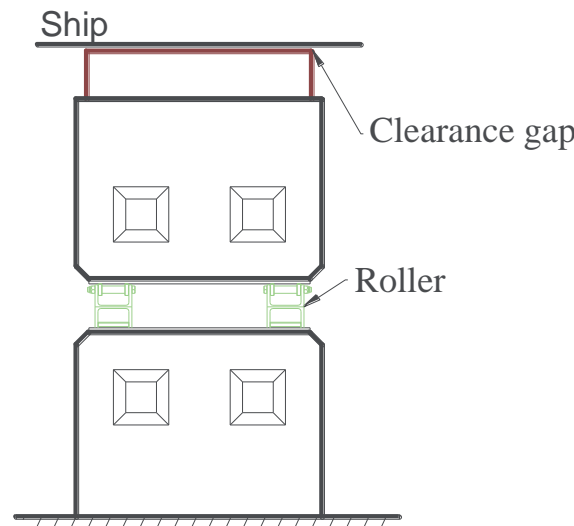

C

Figure 2: Initial version using Type 1 dock blocks

The new arrangement allows hydraulic jacks to be inserted between the blocks. By compressing the softwood capper, the support stools can be removed (Figure 2B), allowing the upper block to be lowered clear of the ship's hull (Figure 2C). Then, using rollers the block is slid to one side. Block replacement is the reverse of the removal procedure.

As Babcock currently owns a large number of the existing (Type 1) blocks, it is preferable to utilise these to prove the new removal system. 


\section{New block removal/replacement mechanism}

\subsection{Stools}

The steel stools support the upper dock block in the new block stack (Figure 2A) and create the necessary space for the installation of the removal mechanism. They are of hollow section so they can be removed using an extracting tool. They are located as close to the block's sides as possible in order to leave free space between the blocks for the jacking system.

\subsection{Removal equipment}

Once the required jacking load has been applied and the softwood compression has created the suitable gap between the two concrete blocks, the stools are removed and two sets of rollers are positioned in their places between the blocks. The jacks are lowered to create a clearance gap with the upper block resting on the rollers. The upper block can then be rolled onto a temporary storage table by hand.

The removal/replacement assembly is shown below (Figure 3).

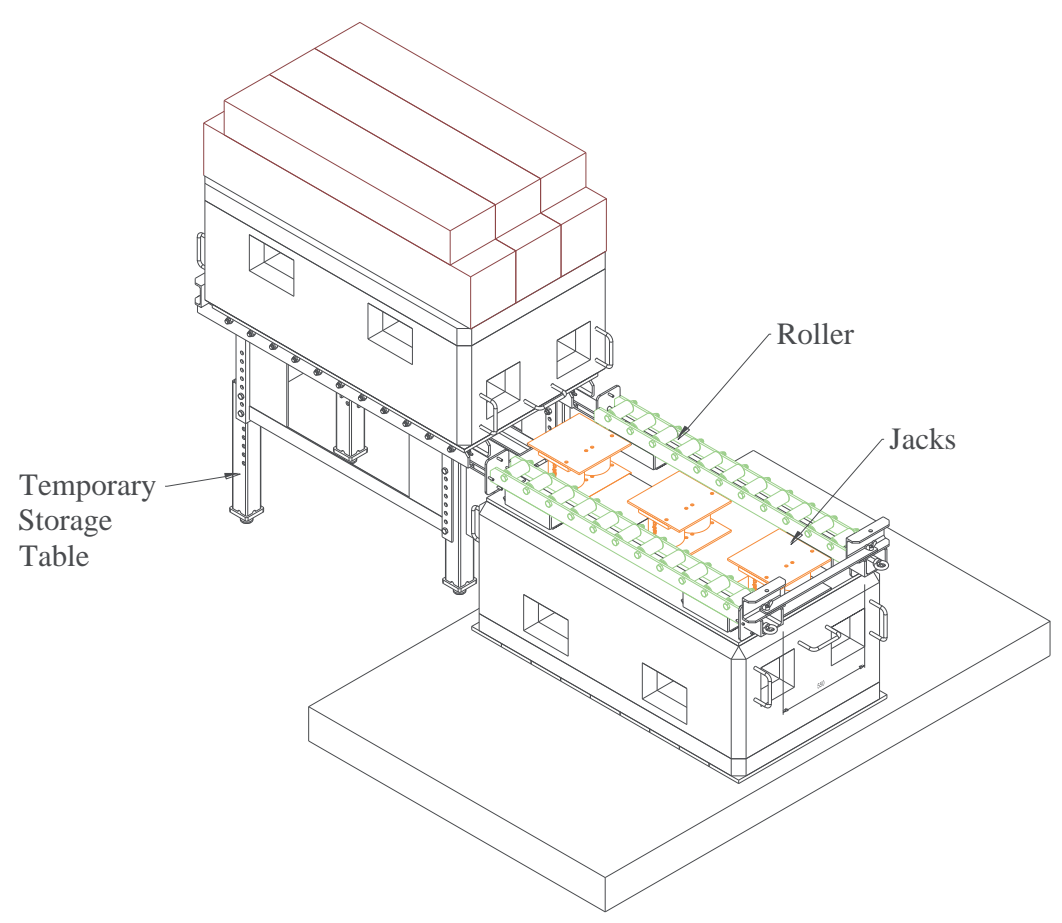

Figure 3: New removal mechanism

It must be noted that ratchet straps, tie bars and other equipment are used to secure the parts in the required place.

The same mechanism is used but the opposite procedure is followed for the replacement of the upper dock block.

- The jacks are positioned on the lower block.

- The rollers are positioned on the lower block and connected to the table.

- The upper block is rolled into position.

- The upper block is jacked up until it creates the required gap for the installation of the stools between the blocks.

- The stools are inserted and the jacks are released.

\subsection{Wood compression tests}

The key element in the removal system is the compression of the softwood, which will create the desired gap between the dock blocks. In order to be able to design the jacking system, it was necessary to assess the compression properties of the timber.

A series of softwood compression tests have been carried out by Babcock and the results have been recorded and analysed. Samples of the standard timber that is used, with thickness varying between $25 \mathrm{~mm}$ and $100 \mathrm{~mm}$, have been tested. The effect of soaking in seawater has been taken into account. 
The results of the various different shaped samples showed a correlation between pressure and strain. The Upper, Lower, Average and Extreme bounds were also plotted to create clear limits of the required pressure. The plots are illustrated below (Figure 4).

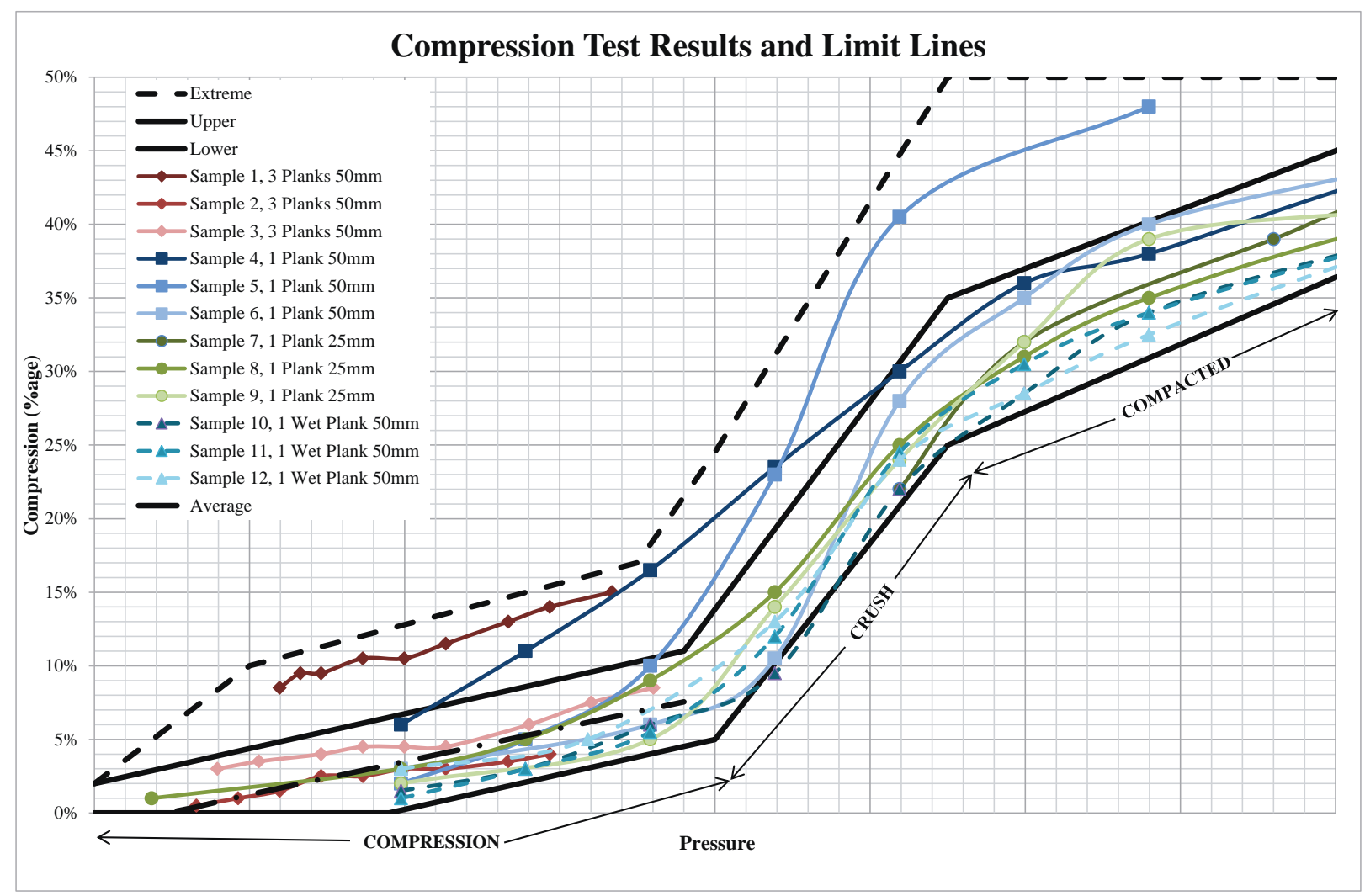

Figure 4: Timber compression test results

It can be seen that the plotted curves have an ' $S$ ' shape, so three modes of response can be defined each having a different rate of strain:

1. "Compression" - Small strain for given pressure.

2. "Crush" - Large strain for given pressure.

3. "Compacted" - Very little change for given pressure.

Although not confirmed during the testing programme, it is assumed that the wood behaves elastically in the compression region and plastically in the crush region.

The testing confirms our instinct to use wood as a material for dock blocks.

In normal use the wood is acting in the initial "compression" region (the extant MoD limit of 165 te $/ \mathrm{m}^{2}$ lies within this region). The wood offers a compliant surface and promotes load-sharing between the blocks.

If something has gone wrong, for example some form of unexpected protrusion, and the block is overloaded, then the wood enters the "crush" region and acts as a fuse. In this mode significant movement can occur without adding significantly more load (Figure 5).

Finally, in the most extreme error scenarios, the wood becomes "compacted" and has enormous strength in compression.

Normal dockings are designed to operate in the "compression" region. The jacking system also operates entirely in the compression region, reserving the crush region as a safety mechanism. 


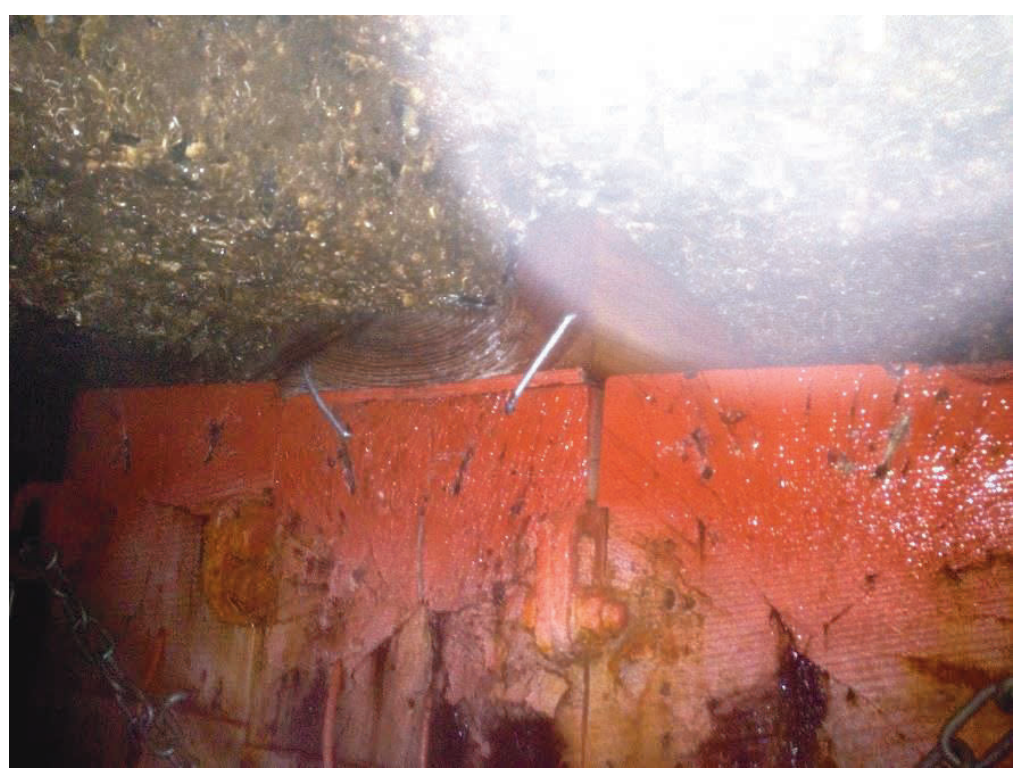

Figure 5: Wood in "crush" region

\subsection{Jacks}

The next step in the investigation was the selection of the jacks. The jacks must produce the required load to overcome the forces acting on the upper block from the ship and the upper block and create compression of the softwood capper in order to create at least a 1-2mm gap for the removal of the stools.

The required capacity of the jacks was calculated according to formula (1):

$$
\text { Required capacity of jacks }=\text { docking load }+ \text { jacking load }+ \text { margin }
$$

The docking load was calculated using the method specified in Ref [1]. This breaks the weight down in to sections between main watertight bulkheads. Overhang weight was then added back in as defined in Ref [2]. The jacking load was calculated using the softwood compression results as described in the previous section. A target compression of $3 \mathrm{~mm}$ is appropriate for most scenarios. As can be seen from the slope of the curves in the results, $3 \mathrm{~mm}$ compression of a typical $50 \mathrm{~mm}$ capper is a large percentage strain $(6 \%)$ and would require a significant force to achieve. In order to alleviate this, thicker cappers were specified. The same compression requirement on a $200 \mathrm{~mm}$ capper is only $1.5 \%$ strain which is much more manageable.

In addition to increasing the depth of the cappers, a potential issue was identified with lightly loaded blocks. From Figure 4 it can be seen that the lower bound of the results shows that some timber exhibits almost no compression at lighter loads. If such a piece of timber was used for an already lightly loaded block there is a risk that it may require a large jacking load in order to achieve the clearance. To prevent this problem, lightly loaded blocks have their timber capper area reduced - thus increasing the static docking pressure, but reducing the potential that the additional jacking load will be excessive.

To select the jacks, the jacking load is added to the docking load for all blocks of all vessels which are anticipated to require docking at Devonport. The loads are checked against the structural capacity of the dock floor, the blocks themselves and the ship. In general, the existing Type 1 dock blocks are the limiting factor as they were not designed to accommodate the point loads imposed by the jacks.

The required jack capacity was defined by all the aspects considered above. For Devonport's ships and dock furniture this capacity was found to be 340 tonnes. With this minimum required capacity, jacks from various manufactures were then reviewed.

Due to space constraints, the number of options was very limited. Space constraints include not only the plan area available, but more challengingly the need for a large stroke when compared to the jack's closed height. The required stroke is made up of the following elements (from top to bottom): Margin to avoid jack overextension, softwood compression, softwood de-compression on unloading, block lowering distance (i.e. clearance for removal), jack removal clearance (with block on rollers), lower margin.

For Devonport, six jacks of 60 tonnes with a $50 \mathrm{~mm}$ stroke were found to be the best solution from a geometry point of view. These jacks were customised and de-rated to 37 tonnes (220 tonnes for six jacks) in order to prevent overloading the existing Type 1 concrete blocks. The jacking assembly is illustrated below (Figure 6). 


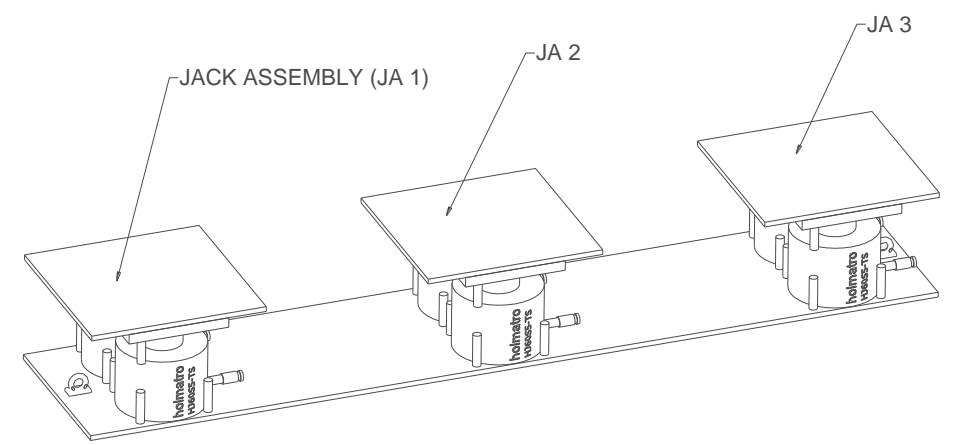

Figure 6: Jacking assembly

\subsection{Operational Experience}

The method described above has already been successfully employed on more than five vessels in Devonport.

The most significant benefit is the large saving of time compared to the traditional method of dock block removal. Where the traditional method could take anything between half a day for an "easy" block and up to two weeks for a particularly troublesome block; this system allows block removal in a few hours.

A further benefit over the traditional method is that blocks can be replaced at very close to their original loading. Using the traditional method, the blocks could not be hardened up fully. This resulted in blocks which were later in the removal sequence becoming progressively more loaded, making them even harder to remove.

In some cases, mostly in way of skeg areas, the calculated block loading is in excess of the capacity of the Type 1 concrete blocks when supported in the manner shown for jacking. Currently this is overcome by using the Type 1 blocks in the traditional manner. This provides virtually full uniformly distributed load over the full plan area of the blocks and therefore brings the loading within the capacity of the Type 1 blocks. Removal is by traditional means. It is only necessary for a small number of blocks as shown in the Table 1 below.

Table 1: Traditional and jack-able dock blocks

\begin{tabular}{cccc}
\hline $\begin{array}{c}\text { Vessel } \\
\text { Class }\end{array}$ & $\begin{array}{c}\text { Total No of Stacks } \\
\text { Required } \\
\text { in Prep }\end{array}$ & $\begin{array}{c}\text { No of jack-able } \\
\text { stacks using } \\
\text { Type 1blocks }\end{array}$ & $\begin{array}{c}\text { No of traditional } \\
\text { stacks }\end{array}$ \\
\hline Ship 1 & 60 & 60 & 0 \\
Ship 2 & 70 & 48 & 22 \\
Ship 3 & 98 & 85 & 13 \\
Ship 4 & 126 & 66 & 60 \\
Ship 5 & 154 & 119 & 35 \\
\hline
\end{tabular}

Whilst only a small number of blocks are affected, they are the higher loaded blocks and are therefore the most likely to be time consuming to remove. The solution to this, as per the title of this paper, is "super dock blocks", which have a higher load capacity sufficient for the docking load and the jacking load.

Before progressing to the super blocks themselves, other operational experience has been gained from the first three vessels that have been docked using the jack-able blocks:

The introduction of hydraulic jacks to a task which has, until now, been carried out by Shipwrights with hammers and chisels has led to some teething troubles. Difficulties have been experienced with incorrect assembly of hydraulic components, inappropriate storage and harsh environmental conditions. Most of these issues have been resolved through training. However, those due to the harsh environment must not be underestimated. This includes very aggressive corrosion and overspray from blasting activities.

Another interesting element of the feedback concerned the positioning of ancillary equipment such as the jacks and the temporary support tables. Although lifting aids were provided for this equipment, in practice the equipment was often positioned by hand. This resulted in feedback that the equipment was too heavy. Also, minor damage occurred (for example the screw-in levelling feet of the temporary support table became bent 
when it was dragged across the dock floor). The root cause of all of these comments is that the lifting aids provided were not easy enough to use. It is apparent that more effort should have been directed towards the manual handling aspects of the design. During the design period, a selection of end users were consulted. However, it was only a small group and shortly afterwards the management structure of the dockyard was changed and it was a different group who became the actual end users. With hindsight it would have been beneficial to gather the views of a wider population of possible end users.

However, as the number of the dockings which use the new removal / replacement system is growing, more operational experience is recorded in an LFE (Learning From Experience) register.

Items from the LFE register are regularly incorporated into updates of the operating and maintenance manual (OMM) for the dock block removal/replacement equipment and the Super Dock Blocks. The OMM is used in the training of the SQEP (Suitably Qualified and Experienced Personnel).

\section{Super Dock Blocks}

Using existing equipment, block loads of up to 220 tonnes can be accommodated. However, during the docking of some ships loads in region of 340 tonnes have been predicted when removing blocks by jacking. A new stronger dock block would allow all the blocks to be removed quickly and efficiently during a normal docking period.

Another consideration towards the design and build of higher strength dock blocks is the requirement of docking ships in deep or damaged condition due to an emergency docking.

The performance of hydrostatic testing of tanks during the docking period has also been a subject for discussion in Devonport in recent years. Currently this is carried out afloat and as such, the activity may lay on the critical path for return of the ship to service. New dock blocks could withstand the high loads from the full tanks and facilitate the testing in dock.

On starting the investigation into super blocks, the option of reinforced concrete blocks was preferred due to their corrosion resistance, general toughness and good operational experience with the Type 1 blocks. It has been found that in order to meet the demand for loads of up to 400 tonnes, the new blocks would consist of a prohibitive amount of reinforcing bars. This issue directed the investigation to a dock block made of steel only.

\section{Design constraints/requirements}

As a new efficient method of removal/replacement of dock blocks has already been designed and put into use, the most significant requirement for the new blocks is to use the same mechanism. It constrains the super blocks to the same dimensions and similar weight as the Type 1 dock blocks.

Each new block stack will be similar to Type 1 stack, i.e. it will comprise of two super blocks with removable spacers. Therefore the super dock blocks must have a robust and plane upper and lower surface to facilitate stacking and other forces exerted on them during the block removal process.

The adverse conditions from the harsh working environment have already been highlighted. The super blocks need to be capable of withstanding the repeated submergence in sea water, exposure in overspray and other contaminants (oil, solvent, etc.) and have a reasonable resistance to damage under shot blast operations. Previous experience with steel dock furniture has informed the design of the new blocks, particularly for aspects such as paint systems, inspection and maintenance.

A further significant aspect in the super block design is handling. Provisions are required for the easy and safe handling of the blocks using dockside cranes, mobile cranes, forklifts and side loaders.

The blocks have been designed to withstand normal handling and usage for a minimum of twenty-five years. An open and accessible structure will allow for the necessary inspection and re-preservation throughout its life cycle.

As part of the design of the super dock blocks, several options were considered with respect to the introduction of the new blocks. The factors considered were the docking loads, the annual capital cost and the ongoing project labour/maintenance cost. These are outlined below:

- All blocks to be Type 1 blocks: This option has been discounted as project labour cost for the block removals remain high.

- All Type 1 dock blocks to be replaced by super dock blocks: This option has a very high initial capital cost and a high through life cost.

- Supplement existing Type 1 dock blocks with super blocks: This option provides the lowest capital and through life cost. It will reduce project costs without requiring excessive capital outlay. If desired super block manufacture can be used as a stand by job for the main factory.

According to the study described above, the new system must integrate with the existing system (Type 1 blocks) as in any one dock prep, only a small number of the stacks will use the new super blocks. Examples showing the number of the required Type 1 and super blocks can be found in Table 1 . 
The original design of the Super Dock Blocks was driven by the end user (the Head of Amphibious outputs) in collaboration with the Health and Safety department.

A number of internal safety reviews have been carried out during the design of the blocks in order to define and eliminate the risks of the utilisation of the Super Dock Blocks in dry docking.

Both the Super Dock Blocks and the original Type 1 blocks require inspection before each use. This is typically carried out during the build of the dock prep. It is anticipated that the Super Dock Blocks will be experience many fewer rejected blocks than the concrete Type 1 blocks. With a corrosion allowance of $5 \mathrm{~mm}$ per face $(10 \mathrm{~mm}$ total) the anticipated lifespan of the Super Dock Blocks is 25 years.

\section{Dimensions and Loads}

Taking into account the design requirements and constraints, the main dimensions for the super blocks and the design loads are summarised below. Figure 7 shows the super block and a typical new block stack.

Stack height (2 blocks plus spacer stools/jacks)

Width

Length

Max Weight (for single block)

\section{Load Carrying Capacity}

Max overall load

Max transferred ground load
$1.7 \mathrm{~m}$

$1.0 \mathrm{~m}$

$1.8 \mathrm{~m}$

4 tonnes

400 tonnes

222 tonnes $/ \mathrm{m}^{2}$
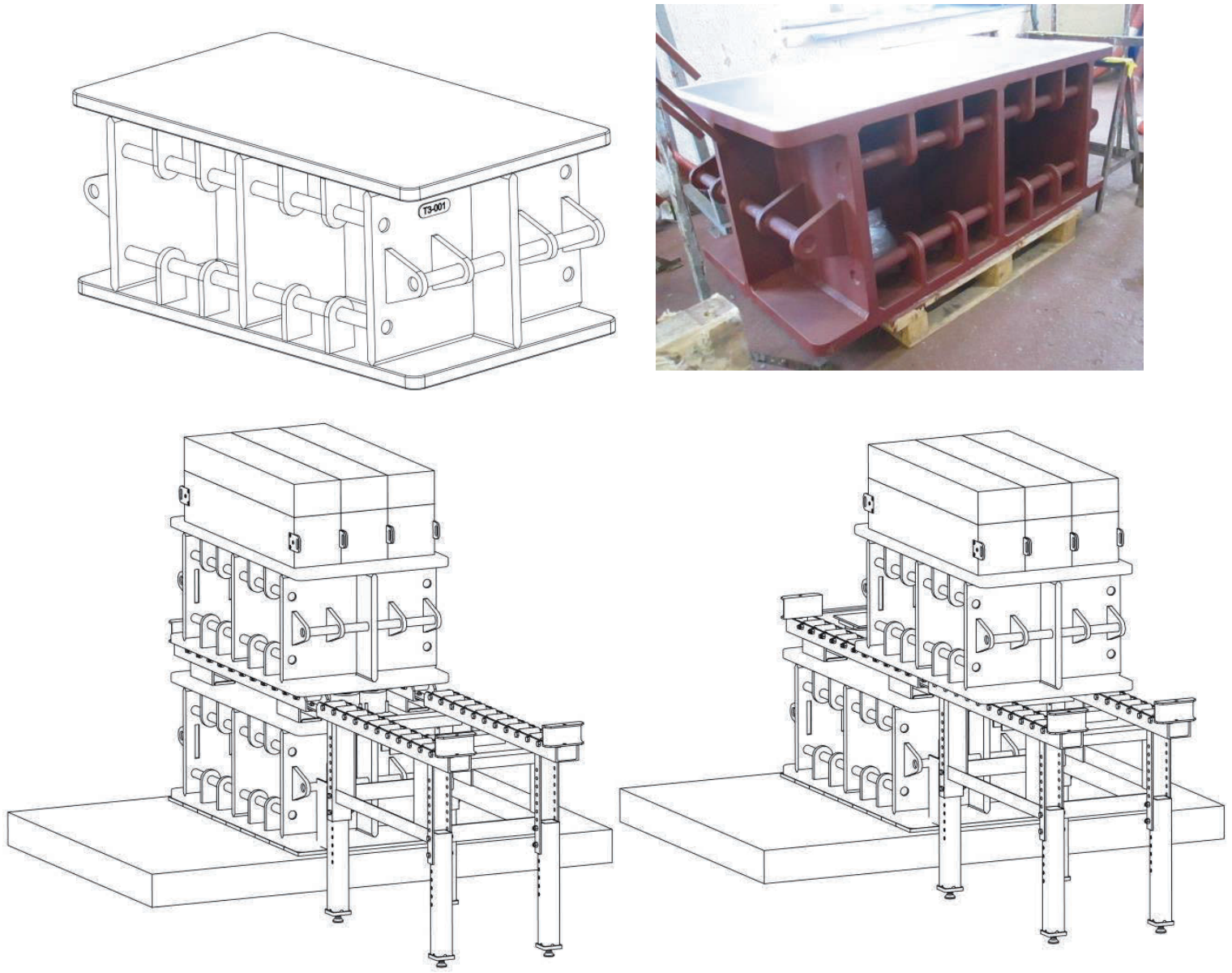

Figure 7: Super dock blocks 


\section{Conclusion}

The development of an approach to remove dock blocks by jacking, rather than by traditional methods has enabled Babcock to reduce the cost and time associated with the inspection and re-preservation of the full extent of ship's outer bottoms. This provides a benefit in the refitting of ships and also allows all fouling to be removed; thus improving the ship's speed or fuel efficiency.

An initial approach has been developed largely making use of existing dock furniture. This approach has required research and development into the properties of timber cappers. It has also required some intricate design which has been totally reliant on a sound knowledge of realistic tolerances in a heavy engineering environment.

The initial approach has, over the course of its use on ship dockings, been demonstrated to be successful. Babcock is now taking this initial success forward: The design of stronger super blocks will extend the use of the technique from approximately $75 \%$ of blocks to $100 \%$ of blocks on ships docked at Devonport Royal Dockyard. Babcock has applied for a patent to cover the technology outlines in this paper.

\section{Acknowledgements}

In addition to the authors, many Babcock personnel at Devonport, both in design and production, have contributed to the development of the jacking system and the super blocks. Their work has made the final system possible.

\section{References}

1. SSCP 23, Volume 1, Original 12.89 - 'Design of Surface Ship Structures', pp 4.11-4.12

2. Lloyd's Register - 'Rules and Regulations for the Classification of Naval Ships', January 2015. Volume 1, Part 3, Chapter 5, Section 10.4.8

\section{Bibliography}

1. The Economic Influence of the Development in Shipbuilding Techniques 1450-1485, J Dixon 1954

2. The Dry Dock Code, W Brook-Hart, R Smith \& G Skinner, 2008 